
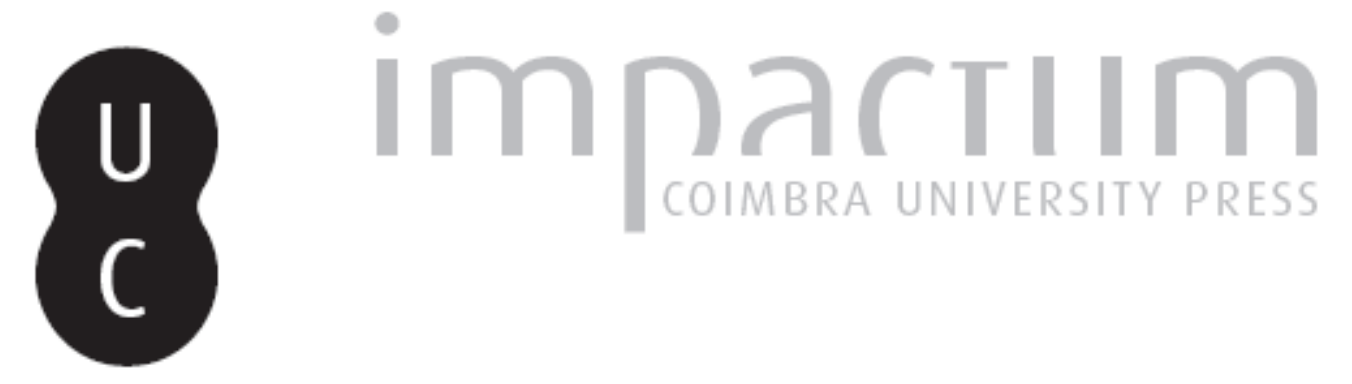

\title{
[Recensão a] Nicolas Grimaldi, Socrate le sorcier
}

\author{
Autor(es): Gaulin, Morgan
}

Publicado por: Imprensa da Universidade de Coimbra

URL persistente:

URl:http://hdl.handle.net/10316.2/42231

DOI:

DOl:https://doi.org/10.14195/2183-4105_6_7

Accessed : $\quad$ 26-Apr-2023 10:15:10

A navegação consulta e descarregamento dos títulos inseridos nas Bibliotecas Digitais UC Digitalis, UC Pombalina e UC Impactum, pressupõem a aceitação plena e sem reservas dos Termos e Condições de Uso destas Bibliotecas Digitais, disponíveis em https://digitalis.uc.pt/pt-pt/termos.

Conforme exposto nos referidos Termos e Condições de Uso, o descarregamento de títulos de acesso restrito requer uma licença válida de autorização devendo o utilizador aceder ao(s) documento(s) a partir de um endereço de IP da instituição detentora da supramencionada licença.

Ao utilizador é apenas permitido o descarregamento para uso pessoal, pelo que o emprego do(s) título(s) descarregado(s) para outro fim, designadamente comercial, carece de autorização do respetivo autor ou editor da obra.

Na medida em que todas as obras da UC Digitalis se encontram protegidas pelo Código do Direito de Autor e Direitos Conexos e demais legislação aplicável, toda a cópia, parcial ou total, deste documento, nos casos em que é legalmente admitida, deverá conter ou fazer-se acompanhar por este aviso.

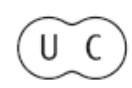




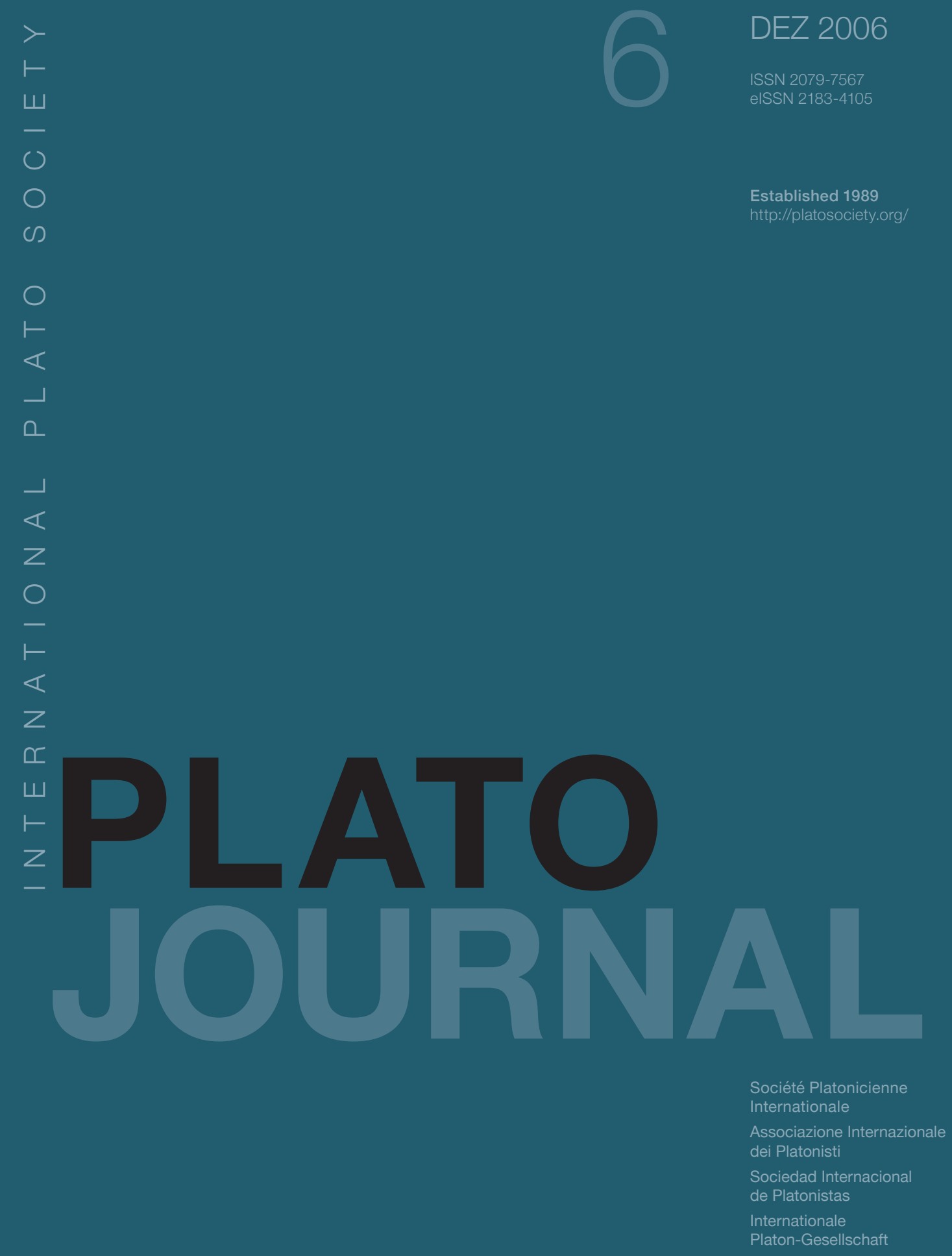


Nicolas Grimaldi, Socrate le sorcier, Paris, PUF, Perspectives Critiques, 2004, 125 pages.

Une des idées les plus fructueuses de la philosophie socratique consista en une remarque suivant laquelle il est impossible de traiter l'âme comme on traite le corps. L'âme requiert des mots, et le philosophe est celui qui a la responsabilité d'élaborer une « thérapeutique de la parole ». Cette médecine, Nicolas Grimaldi démontre qu'elle fait de Socrate un sorcier en tout point semblable au chaman. Mais celle-ci témoigne aussi, selon nous, d'une visée désignant le fait que, tel que lui fit remarquer Calliclès dans le dialogue du Gorgias, Socrate ne fut pas toujours de bonne foi. En d'autres mots, si le dialogue socratique fut toujours fixé, tel que le rapporte Nicolas Grimaldi, sur la seule découverte de la vérité, il est tout aussi vrai qu'il laisse transparaître à certains moments un désir caché de dominer. Ainsi, la formule qu'utilise l'auteur pour caractériser le sophiste - « le sophiste est comme le loup: c'est un prédateur. Son gibier, c'est l'autre » (31) - peut tout aussi bien s'appliquer à Socrate. À l'aide de l'instrument nommé " rhétorique », les sophistes, en effet, cherchèrent à dominer le monde. Or, selon la thèse de Nicolas Grimaldi, Socrate propose l'exact contraire; c'est le détachement qui doit être recherché par le philosophe afin d'arriver à accéder à l'essence éternelle des choses. Une remise en question de ce détachement par Calliclès est pourtant parvenu à remettre en question le désintéressement affiché par le philosophe.

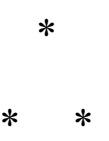

Notons que l'ouvrage intitulé Socrate le sorcier n'est pas un commentaire dédié strictement à un public de spécialistes. Il s'agit d'un essai philosophique rédigé dans l'esprit de pouvoir s'adresser aussi aux non-initiés. Les notes de bas-de-page y sont réduites au minimum et aucune bibliographie n'y figure. Nicolas Grimaldi, d'abord reconnu comme un spécialiste de Descartes - rappelons qu'il publia L'expérience de la pensée dans la philosophie de Descartes (1978), Six études sur la volonté et la liberté chez Descartes (1988), et Études cartésiennes. Dieu, le temps, la liberté (1996) -, n'en est pas à ses premières armes dans le domaine de l'essai, le Bref traité du désenchantement (1998) et L'homme disloqué (2001) ont retenu l'attention de la scène philosophique.

Dans Socrate le sorcier, la thèse formulée par Nicolas Grimaldi définit la figure légendaire de Socrate comme celle qui correspond aux quatre caractéristiques du chaman telles qu'elles ont été énumérées par Mircea Eliade dans l'essai intitulé Le chamanisme et les techniques archaïques de l'extase (Paris, Payot, 1951, 1968²). Selon la typologie établie par Eliade, le chaman est ce sorcier qui fait office de guérisseur, qui travaille à redéfinir l'identité des individus auquel il s'adresse, qui est choisit par les dieux et, enfin, qui a connu l'extase à au moins une reprise. Socrate, tel que se le représente Nicolas Grimaldi, est lui aussi un guérisseur. L'auteur note, en effet : " car ce qui fait de Socrate un sorcier, c'est d'abord qu'il est un guérisseur » (11). Mais comment est-il possible de réconcilier ce chamanisme avec la discipline rationnelle nommée " philosophie »? Grimaldi rappelle que Socrate soutenait que le philosophe devait enseigner autant par sa vie que par ses théories (sa mort fut en ce sens l'incarnation la plus radicale de son enseignement). Socrate croyait aussi que la vérité pouvait changer la vie d'un homme, et parce qu'il s'attachait à servir la 
vérité, et non ses propres intérêts, il se rapprochait effectivement du chaman, qui se met volontairement en retrait de la société, et se détache du monde. Socrate était forcé, admettra-t-on avec Grimaldi, de favoriser le détachement puisqu'il considérait que la vérité n'était pas acceptée par les assemblés des citoyens. Or, en se détachant de la sorte, il arrivait à se tenir, par le fait même, à l'écart de l'injustice engendrée par le mensonge. Mais comment éviter que le philosophe ne retombe, à un moment ou à un autre, dans l'empire du mensonge? Nicolas Grimaldi rappelle que la solution socratique à ce problème consista à faire en sorte que les hommes cessent de craindre la mort. Socrate enseigna aux hommes que leur vie ne tenait en réalité qu'à ce qu'ils en faisaient, et non à la manière dont ils se la représentaient. Afin de convaincre les athéniens de ces deux postulats, Socrate se servit de la philosophie comme d'une thérapie, c'est-à-dire comme d'une logique séduisante ayant pour fonction de produire, chez ses interlocuteurs, un « ensorcellement » tel que l'a rapporté Ménon, ou encore un « envoûtement », comme ce fut le cas pour Alcibiade (9). Mais voilà, pour guérir l'homme de la peur de la mort, il fallut d'abord le libérer du monde car, rappelle Grimaldi, celui-ci, toujours changeant, demeure « innommable » et " inexprimable », et nous conduit à désespérer de nos propres capacités à le comprendre. Aussi, le langage de la thérapie philosophique n'a pas comme objet de rendre compte du réel, mais fait plutôt en sorte que ce qu'il énonce devienne le réel. C'est ce renversement qui, selon l'auteur, fait du philosophe un sorcier. Dès lors, la parole n'a plus à peiner devant le chaos qu'est le monde, et la vérité ne concerne plus alors que le seul langage. Grimaldi note que ce que nous avons l'habitude de traduire par « Idées » ou « principes » renvoi à l'expression grecque «tous logous », désignant les mots dont nous nous servons pour traduire nos pensées. Nicolas Grimaldi est alors en mesure de démontrer que la magie de Socrate repose sur la correspondance des Idées aux mots, et non plus des mots aux choses réelles, ce qui permet au philosophe de remonter des paroles aux Idées éternelles et immuables. De cette manière, Socrate est arrivé à neutraliser la peur de la mort en démontrant le postulat de l'immortalité de l'âme (70), mais il engendra, simultanément, la possibilité d'une vie après la mort; le sentiment de la peur ne se trouvait donc que déplacé vers un au-delà. La thérapie philosophique devait ainsi proposer un nouveau mythe. Celui-ci expliqua aux hommes que la vie incorporelle surpasse de loin la corporalité, la vie en ce monde. Nicolas Grimaldi explique que ce mythe est venu compléter la logique philosophique en y ajoutant un " envoûtement », un ensorcellement supplémentaire, permettant à Socrate de justifier et de donner une fondation à la thèse centrale de sa philosophie suivant laquelle le semblable ne peut tirer de bonheur que de son semblable (88). Les désirs de l'âme ne peuvent jouir que de ce qui est, comme elle, éternel. L'âme humaine, ne cessant de désirer ce qui est durable, se détourne progressivement du monde, qui ne fait que lui proposer des simulacres éphémères. La magie socratique consista à persuader les âmes souffrantes que la mort n'est pas notre ennemie, et qu'elle n'est autre qu'une « délivrance » annonçant une vie meilleure.

Socrate expliqua à ses interlocuteurs qu'il est vain de désirer ce qui est changeant et temporel. Mais, de notre point de vue, c'est en ce point précis qu'il nous semble important, et ce contrairement à Grimaldi, de mener une critique de Socrate. En ce sens, le personnage de Calliclès, auquel Grimaldi n'accorde, selon nous, pas assez de crédit, a su reprocher à Socrate de se « déguiser » et de s'emmurer derrière un système afin de ne pas souffrir de la peine résultant d'avoir espéré en ce qui peut, à tout moment, nous décevoir. Suivant Calliclès, Socrate aurait eu peur de s'engager dans le monde parce qu'il aurait craint d'être 
blessé (par exemple, il « drague » tous les jeunes athéniens, mais ne couche avec aucun). Sa sorcellerie l'aurait, de la sorte, protégé de ses propres sentiments. Combien de passages des dialogues de Platon ne rapportent-t-ils pas Socrate mettant en garde ses interlocuteurs contre la souffrance et la peine que leur réserve le monde ? Le philosophe socratique pourrait, en cela, être comparé à un enfant qui ne peut supporter d'être soudainement privé d'un plaisir dont il a l'habitude, et nous persuade que, parce que nous risquons d'être déçus d'une telle privation, il nous faut oublier le monde, nous en guérir. Il serait alors possible d'objecter à la philosophie de Socrate que ce n'est pas parce que le plaisir ne dure pas qu'il n'est pas plaisant, et n'est donc pas digne d'être assumé. À ce propos, Calliclès a, selon nous, habilement su opposer au philosophe ce que nous nommons, pour notre part, la « persévérance du désir », et sa prise en compte par la pensée. À cette sorcellerie socratique, construite contre le monde, Calliclès, dans le dialogue du Gorgias, a résisté et, de la sorte, est arrivé à souligner un caractère de la personnalité de Socrate que Grimaldi n'a pas relevé; "Voilà bien longtemps que je t'écoute, Socrate, et que j'acquiesce à tes propositions, en me disant que, si l'on s'amuse à te faire la moindre concession, tu la saisis avec une joie d'enfant » (Platon, Gorgias, LIV). C'est cet enfantillage qui nous semble être le plus douteux, et le moins propice à conduire un dialogue de portée réellement thérapeutique, et dont Grimaldi ne rend point compte. Mais que signifie au juste cet enfantillage ? Socrate définissait l'âme comme étant éternelle parce qu'elle est de nature immatérielle (70-71), nature qui lui est révélée à l'occasion de la réminiscence (67-69), cette faculté qui consiste à reconnaître la vérité, c'est-à-dire les Idées éternelles, les essences des choses entrevues par l'âme avant qu'elle ne se fixe à un corps. La réminiscence fait en sorte de nous détacher de la matière pour nous rappeler notre origine dans l'éternité, et le dialogue socratique vise à produire, en nous, l'effet momentané de cette reconnaissance. De la sorte, l'âme se trouve ré-acheminée vers son origine. C'est de ce retour vers le point de départ de toutes choses dont Calliclès nous parle alors qu'il discute avec Socrate, et qu'il souligne que la dialectique que propose la philosophie à ses interlocuteurs risque de confondre l'origine à l'enfance. L'enfantillage, qui est aussi le propre des sophistes, et de tous ceux qui tirent du plaisir à dominer les autres, constitue, aux yeux de Calliclès, un glissement dangereux vers l'obsession que nous posséderions tous secrètement de retourner vers l'enfance, enfance qui équivaudrait à une « forme » de l'unité originaire. En acceptant la logique de Calliclès, telle que l'a rapportée Platon dans les dialogues, et en prenant nos distances par rapport aux propos de Grimaldi, il est possible d'affirmer que le sensible n'est pas une illusion, que la matière n'est pas non plus une idée abstraite de quelque savant; la maladie en serait d'ailleurs l'exemple extrême, rendez-vous avec le réel que personne ne peut manquer. Socrate, si l'on accepte de prendre au sérieux ce qu'en dit Calliclès fut, au fond, un " enfant sérieux », un dominateur sophistiqué, qui eut, de surcroît, le talent d'adapter ses discours aux orateurs auxquels il s'adressait (Phèdre). À partir de ce point de vue, Socrate établit donc le dialogue philosophique davantage pour avoir raison de ses contemporains que pour arriver à savoir de quoi, au juste, il était question et, surtout, de quoi il aurait dû être question dans la vie de la Cité.

Une toute dernière remarque, d'ordre historique, doit être adressée à cette thérapie de la parole, à propos de laquelle il faut bien dire que Socrate n'est en rien l'inventeur. Empédocle avait déjà été loin en ce domaine. Fils de Méton, il vécut d'environ 492 à 432 avant J.C. et fut philosophe, médecin, orateur et thaumaturge. Une légende le rend 
responsable d'avoir libéré la ville de Sélinonte de la malaria. Selon la philosophie d'Empédocle, le monde est triste; il est comme une « caverne recouverte d'un toit » (Empédocle, fragment 120, in Jean-Paul Dumont, Les Présocratiques, Paris, Gallimard, 1988). Il s'agit, évidemment, d'une image renvoyant à l'allégorie de la caverne que rapporta par la suite Platon dans la République. Le fragment 112 d'Empédocle rapporte une « chute du haut du bonheur »; la vie humaine est donc, selon lui, nécessairement misérable, et les hommes sont condamnés à souffrir de " maux cruels ». (Empédocle, fragment 112) Afin de guérir les êtres humains de ce monde, Empédocle n'hésitait pas, et ce bien avant Socrate, à croire au « miracle de la parole », et à apostropher ses contemporains pour leur apprendre la vérité sur leur propre vie, les enjoignant à cesser de croire en ce monde. Une autre référence des dialogues de Platon nous apprend que les Thraces avaient, eux aussi, développé l'idée d'une guérison par la parole. Socrate les a connus au cours d'une campagne militaire, et s'y réfère alors qu'il discute avec Charmide. Citant un médecin Thrace avec qui il s'était entretenu, il se rappelle que ce dernier lui avait dit que les véritables remèdes de l'âme ce sont les incantations, et que celles-ci consistent en certains discours. (Platon, Charmide, 155-157) Socrate n'est donc pas un découvreur en ce domaine, et il serait, à ce propos, pertinent de rappeler certaines thèses pythagoriciennes et orphiques pour s'en convaincre. À ce sujet, Karl Kerényi a accompli jadis un beau travail (« Pythagoras und Orpheus », in Humanistishe Seelenforschung, Munich, 1966, p.20), et plusieurs travaux d'anthropologues ont remis de l'avant la présence de psychothérapies religieuses chez plusieurs peuples primitifs comme les Indiens d'Amérique du Nord.

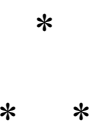

L'essai de Nicolas Grimaldi constitue un ouvrage incontournable des études socratiques parce qu'il innove en comparant l'exercice socratique de la philosophie au chamanisme. Comme le souligna Mircea Eliade, le chaman est celui qui, après avoir été visité par des divinités, se convertit à un autre monde et, de la sorte, s'opposa à l'existence ordinaire des hommes. Ce fut aussi le cas de Socrate, pour qui mourir à ce monde fut, en toute dernière instance, préférable à l'obligation de s'abaisser à prouver sa valeur aux citoyens d'Athènes. L'opposition de Calliclès à Socrate, à laquelle nous avons fait appel, incarne, quant à elle, l'affrontement entre les attitudes d'engagement et de détachement, que Grimaldi n'a fait que mentionner au passage (27), tout en s'attachant, et ce beaucoup plus longuement, à la justification du détachement que formula Socrate (27-31). Prenant au sérieux (ce que, selon nous, Grimaldi n'a pas fait) la critique que Calliclès adressa au détachement socratique, il nous est apparu que la posture du philosophe ne fut pas totalement désintéressée des « affaires humaines ». En cela, il est bien possible que Socrate fut tout près des sophistes, qu'il combattait et qui cherchèrent, selon ses propres mots, à dominer le monde, à se l'accaparer. La distinction qu'il serait alors possible d'établir, de notre point de vue, entre Socrate et ses ennemis, serait que les seconds (les sophistes) tentèrent de se rendre maîtres du monde de l'intérieur de celui-ci, alors que le premier (Socrate) s'est proposé de le contrôler de l'extérieur, à partir du ciel des Idées, et de s'ériger en une cause régulatrice et extramondaine du cours de l'histoire humaine comme si Socrate avait découvert que se connaître soi-même, c'est se dominer, et que se dominer, 
c'est aussi, après tout, dominer le monde. À l'issu de ce brillant essai, il demeure cependant une question non résolue; pourquoi Socrate insistait-t-il tant à faire en sorte de guérir l'âme de ses interlocuteurs ? Et d'abord pourquoi a-t-il tant insisté à convaincre les athéniens qu'ils étaient malades?

Morgan GAULIN

Université de Montréal 